\title{
The Barriers to Initiating Insulin Therapy among People with Type 2 Diabetes in Taiwan - A Qualitative Study
}

\author{
Kwang-Wen Chen ${ }^{1 *}$, Hsu-Min Tseng ${ }^{2}$, Yu-Yao Huang ${ }^{3}$ and Yuh-Jue Chuang ${ }^{4}$ \\ ${ }^{1}$ Division of Endocrinology and Metabolism, Department of Internal Medicine, St Paul's Hospital, Taiwan \\ ${ }^{2}$ Graduate Institute of Health Care Management, Chang Gung University, Taiwan \\ ${ }^{3}$ Division of Endocrinology and Metabolism, Department of Internal Medicine, Chang Gung Memorial Hospital, Taiwan \\ ${ }^{4}$ Graduate Institute of Health Care Management, Chang Gung University, Taiwan
}

\begin{abstract}
Insulin therapy often becomes necessary when oral hypoglycemic agents are no longer effective. However, the rate of initiating insulin treatment is low among people with type 2 diabetes in Taiwan. A qualitative study was done at the diabetes centers of two hospitals and consisted of face-to-face interviews of patients who were reluctant to start insulin treatment. The results of interviews were subjected to Framework Analysis for emergent concepts and category system. There were 15 people (10 women, 5 men) who were interviewed, ages between 46 to 71 years old (mean age 59.7 \pm 7.3 years). We categorized the barriers of initiating insulin treatment as 1) Reluctance of physicians 2) Misconceptions about insulin 3) Low adaptation capacity 4) Needle phobia 5) Psychological insulin resistance. These barriers interfered with decisions to begin insulin treatment. To break these barriers, responsibility and decision-making should be placed upon caregivers for people with low adaptation capacity. Needle phobia should be looked at as a disease entity and addressed with greater patience. The possibility and benefits of insulin treatment should be included early in diabetes education. Physicians should adhere to the guidelines for initiation of insulin treatment and empower patients.
\end{abstract}

Keywords: Barriers to insulin treatment; Type 2 diabetes; Qualitative study

\section{Introduction}

Both the UKPDS and the DCCT have shown that aggressive glycemic control can prevent diabetic complications $[1,2]$. If patients do not achieve glycemic goals, for example with metformin treatment, insulin treatment is necessary [3]. Early intervention with insulin in patients with type 2 diabetes has been shown to result in better outcomes such as recovery of beta-cell function and longer glycemic remission when compared with sulphonylurea [4-7]. The goal of glycemic control is A1c less than $7 \%$. In the US, $55.7 \%$ of patients achieved this in 2003-2004 [8]. However in Taiwan, 32.4\% reached this A1c goal and insulin treatment was used in only $16.9 \%$ in 2006 [9].

Insulin is clearly effective in controlling blood glucose and preventing further diabetic complications. But insulin therapy is not used in most patients even if it is indicated because of several obstacles. The following barriers for initiating insulin therapy have been reported: fear of needle (needle phobia), social stigma, insulin therapy indicates advancing disease, fear of permanent use of insulin, feelings of self failure, inconvenience, loss of personal freedom, fear of side effects such as hypoglycemia, weight gain, or misconceptions about insulin such as causing damage to kidneys or body [10-15]. Peyrot et al. [14], reported that patients rate the efficacy of insulin as low and blame themselves if they have to start insulin therapy. Insulin treatment is also considered to be a social stigma in Chinese culture. Distrust of western medicine and trying traditional Chinese medicine before accepting insulin injection were culture barriers in Canadian Chinese [15]. A low rate of insulin treatment $(16.9 \%)$ in Taiwan indicated that people with type 2 diabetes in Taiwan found it difficult to initiate insulin [9]. In recent years, new insulin analogues and the pen injector have been associated with less hypoglycemia, less weight gain, more flexibility and more convenience for patients, so this might make insulin therapy more acceptable $[12,16]$. To understand the barriers to initiating insulin among people with type 2 diabetes is crucial in Taiwan. We therefore performed this qualitative study in order to understand the barriers of initiating insulin treatment among people with type 2 diabetes in Taiwan and to find possible strategies to overcome these barriers

\section{Methods \\ Interview}

The study took place in diabetes centers of Chang Gung Memorial Hospital and St. Paul's Hospital in Taoyuan County of Taiwan. There were 15 patients who met the criteria of being $>20$ years of age, refusing insulin treatment and A1c $>8 \%$ despite being on 2 to 3 kinds of oral hypoglycemic agent, and were referred to interviewers. Patients who were pregnant or who had problems with communication were excluded. Ethics approval was obtained from the Ethic Research Board of Chang Gung Memorial Hospital and St. Paul's Hospital. The interviews were performed by 3 experienced diabetes educators who were trained by the investigators. The interviewers were instructed to be friendly and non-prejudicial and allowed patients to talk freely. The interviews followed a questionnaire consisting of open-ended questions and free conversation in order to obtain information from the patients about their experiences and internal framework of their value system. All the interviews were recorded with $\mathrm{mp} 3$ recorders and transcribed imme-

*Corresponding author: Kwang-Wen Chen, Division of Endocrinology and Me tabolism, Department of Internal Medicine, St Paul's Hospital, No 123 ChienHsin St, Taoyaun City, Taiwan; Tel: 886-3-3610304; Fax: 886-3-3773373; Email: enjoy.kwangwen@gmail.com

Received November 03, 2011; Accepted November 29, 2011; Published June 08, 2012

Citation: Chen KW, Tseng HM, Huang YY, Chuang YJ (2012) The Barriers to Initiating Insulin Therapy among People with Type 2 Diabetes in Taiwan - A Qualitative Study. J Diabetes Metab 3:194. doi:10.4172/2155-6156.1000194

Copyright: (c) 2012 Chen KW. This is an open-access article distributed unde the terms of the Creative Commons Attribution License, which permits unrestricted use, distribution, and reproduction in any medium, provided the original author and source are credited. 
diately following the interview. The scripts were read and discussed to identify themes and whether further interview was needed. Interviews were conducted in Taiwanese and Chinese, and recorded in Chinese. Field notes were made on body gestures, body language, and vocal intonation of the informants based upon observations made by the interviewers.

\section{Data analysis}

The data were analyzed and interpreted using framework analysis, developed by Ritchie and Spencer [17]. The concepts, category system, associations and explanations derived from the data were compared with the general literature before proceeding with further analysis.

\section{Results}

Demographic information about the 15 participants and their barriers to initiating insulin therapy are summarized in (Table 1). There were 10 women and 5 men with type 2 diabetes who were interviewed. The age range was 46 to $71 \mathrm{yr}$ (mean age $59.7 \pm 7.3 \mathrm{yr}$ ). HbAlc was $8.4 \%$ to $12.5 \%$ (mean $11 \pm 1.3 \%$ ). There were 2 cases (Case 7 and 8 ) that had been briefly treated with insulin in the past. Barriers forinitiating insulin treatment fell into the following categories: 1) reluctance of physicians 2) misconceptions about insulin 3) low adaptation capacity 4) needle phobia and 5) Psychological insulin resistance. Multiple barriers could exist in the same individual and might interact with each other. Each category was identified and defined as follows:

"Reluctance of physicians" is recognized by the following types of statements from patients who attribute the postponing of insulin treatment to physicians or to physicians who did not clearly explain the need for insulin.

"Dr. X did not ask me to use insulin therapy now he meant I would need insulin if I did not improve glycemic control again. (Case 1/M/46 yr)

I refused insulin treatment. He just smiled at that. (Case 2/F/60 yr)

I told Dr. X if you want me to inject (insulin), I will inject. He replied that we will discuss next time. (Case $3 / \mathrm{M} / 52 \mathrm{yr}$ )

\begin{tabular}{|l|l|l|l|l|l|l|}
\hline $\begin{array}{l}\text { Case } \\
\text { No. }\end{array}$ & Sex & $\begin{array}{l}\text { Age } \\
(\mathrm{yr})\end{array}$ & $\begin{array}{l}\text { Duration } \\
(\mathrm{yr})\end{array}$ & $\begin{array}{l}\text { A1c } \\
(\%)\end{array}$ & $\begin{array}{l}\text { Complications and associ- } \\
\text { ated disease }\end{array}$ & Barriers \\
\hline 1 & M & 46 & 9 & 11.9 & chronic hepatitis & 1,5 \\
\hline 2 & F & 60 & 11 & 8.4 & nephropathy, hypertension & 1,4 \\
\hline 3 & M & 52 & 9 & 9.3 & hypertension & 1 \\
\hline 4 & F & 54 & 16 & 9.4 & hypertension & $1,4,5$ \\
\hline 5 & M & 58 & 12 & 11.7 & retinopathy & 2,5 \\
\hline 6 & F & 70 & 9 & 12.4 & neuropathy, & 4 \\
\hline 7 & F & 71 & 20 & 9.8 & retinopathy & 5 \\
\hline 8 & F & 57 & 6 & 11.2 & NA $^{*}$ & 2,5 \\
\hline 9 & F & 64 & 16 & 12.5 & neuropathy & $2,3,5$ \\
\hline 10 & M & 68 & 15 & 10.8 & neuropathy & 5 \\
\hline 11 & F & 65 & 2 & 10.6 & NA $^{*}$ & 4,5 \\
\hline 12 & F & 58 & 10 & 12.5 & retinopathy & 3 \\
\hline 13 & F & 62 & 10 & 11.4 & NA $^{*}$ & 2,3 \\
\hline 14 & F & 51 & 8 & 12 & NA $^{*}$ & 1,3 \\
\hline 15 & M & 65 & 10 & 11 & Nephropathy, retinopathy $^{*}$ & 1,5 \\
\hline
\end{tabular}

$N^{*}$ : not recorded. Barriers ${ }^{* *}: 1=$ reluctance of physicians, $2=$ misconceptions about insulin, $3=$ low adaptation capacity, $4=$ needle phobia and $5=$ psychological insulin resistance

Table 1: Clinical characteristics and barriers of insulin treatment of the people with type 2 diabetes who reluctant to insulin therapy.
Doctor did not tell me anything about the benefits of insulin. I refused and he never insisted upon it. (Case 4/F/54 yr)"

"Misconceptions about insulin" is when fear is due to misinformation about insulin. They quote advice or information received from family, friends, relatives and neighbors. Representative statements from patients were as follows:

"Insulin will damage your nerves. My elder sister was treated with insulin and then went on hemodialysis within 6 months. (Case 5/M/52 yr)

Insulin will damage kidney and liver and will lead you to hemodialysis. (Case 10/M/68/ yr)

Insulin has no effect on blood sugar. (Case 9/F/64 yr)

My sister said we have to inject into blood vessels. (Case 14/F/51 yr)

Injection will be addictive; I was told that insulin will cause skin necrosis. (Case 8/F/57yr)"

The individuals with "low adaptation capacity" were identified by their inability to inject themselves, dependent lifestyle, and poor physical condition. Their statements were as follows:

"Because I don't know how to inject and I am afraid of it, let me go naturally. No one could inject for me. (Case 13/F/62 yr)

I have trouble to inject and I don't want to bother others. (Case 9/F/64 yr)

If someone can inject for me in public clinics that will be fine, injecting by myself is impossible. (Case 14/F/51yr)"

"Needle phobia" was the category for those patients who expressed fear of needle and this was accompanied by body language. The patients would also describe how they were fearful of blood tests. Their responses and statements were:

"I was scared when my doctor mentioned injection of insulin. I am scared and dare not watch my brother-in law inject. (With facial expression of fear and body tremor) (Case $12 / \mathrm{F} / 58 \mathrm{yr}$ )

My heart is pumping. I don't want to inject. I am scared of needle injection. (Case 6/F/70 yr)

Even blood test for sugar is very awful and painful. (Case 4/F/54 yr)

When I am waiting for blood test my heart beat is very fast. I am afraid of injection since very young. I would rather take oral medication and no injection. (Case 2/F/60 yr)"

"Psychological insulin resistance" is a complex category which might be due to social stigma, self blame, feeling of self failure, general burden and anxiety with insulin treatment, and fear of adverse effects of insulin. These are statements given:

"If I receive insulin treatment, it means I am very sick, there will be no other medication that could be effective, and I will die soon. (Case10 /M/68yr)

Oral medications will become less and less effective; insulin injection will become more and more. (Case1 /M/46 yr)

Insulin injection is the last resort; My younger brother said that insulin will kill him. (case 5/M/58yr)

It will be life-long (permanent) use of insulin and it is better is not to use it. I worry about the sequelae of insulin. (Case 7/F/71yr) 
My family did not want me to inject, it will be addictive. The skin will be necrotic, indurated. I feel stressed, it is very inconvenient to me. (Case 8/F/57 yr)

I heard that insulin is not effective, and insulin will cause fainting (hypoglycemia coma), It will be life-long injections. (Case 11/F/65 yr)

I heard it will cause tremor, convulsion (hypoglycemia), I feel stressed and inconvenienced, my family asked me not to inject and I also think so. (Case 9/F/64 yr)

I do not have time and I will forget to inject it. (Case 4/F/54yr)

Injection will become permanent, oral medication is better. (Case $12 / \mathrm{F} / 58 \mathrm{yr}) "$

\section{Discussion}

This is a qualitative study to interview the people with type 2 diabetes in Taiwan who refused to initiate insulin treatment. From the results, we categorized the major barriers to initiating insulin treatment as 1) reluctance of physicians 2) misconceptions about insulin 3) low adaptation capacity 4) needle phobia and 5) psychological insulin resistance. The barriers that we identified in this study are common to many patients with type 2 diabetes as previously reported, but other barriers such as fear of weight gain was not found. This may be due to differences in study design, weight gain usually resulting from a longer term of insulin treatment whereas most of our patients had no experience with insulin treatment. We suggest that this classification may be useful in clinical practice, directing physicians to identifying the barriers to initiating insulin treatment and encouraging communication with each other. However, multiple barriers may coexist in a single individual and interaction between barriers is common. Thus it is important not to categorize the patients having only a single barrier.

Low adaptation capacity is a unique category in our classification. This barrier is commonly seen in elderly people who are dependent on others. The elderly have more health problems, find technology difficult, have failing eyesight, and may be unintentionally noncompliant $[18,19]$. This barrier could be overcome only if families or care-givers take the full responsibility for insulin treatment.

Needle phobia is reported to be a formal disease entity, approximately $10 \%$ of the population is affected, but it is always neglected [20]. Patients will become very frightened and stressed when insulin treatment is raised and this may be unrecognized. When a patient is known to have needle phobia, the physician and nurse should be more careful and patient. To reassure and desensitize a patient with this condition, a careful, thorough explanation and demonstration of the needle and injection is recommended. The use of topical creams containing local anesthetics, or nerve gate-blocking methods such as rubbing or pinching during a needle-stick could be helpful [20]. But more knowledge is required before starting insulin treatment in people with needle phobia.

Misconceptions about insulin are very common in people with or without diabetes in Taiwan. Our participants believed insulin would impair renal, liver, and nerve function and they would become addicted. Some of our participants also had no idea of the timing and techniques of insulin treatment and even the benefits of insulin. Patients are not aware that insulin may not only decrease blood sugar, but also improve beta cell function. In clinical practice, education about insulin treatment is done at the time of shifting to insulin therapy. Better understanding of insulin treatment, especially the benefits of insulin could help people to make a better decision.
It has been reported that insulin treatment is often delayed by physicians because they overestimated injection-related worries and fear of severe hypoglycemia with insulin treatment [21]. In this study, we found that some patients thought that postponing insulin was due to their doctors not insisting that insulin treatment be started. They were waiting for the effect of new oral medications. It had been reported that the rate of insulin treatment decreased because of new oral hypoglycemic agents such as thiazolidinediones and glinides [9]. Physicians should offer the algorithm of diabetes treatment to patients and empower them to set their own goal and choose their own mode of treatment.

Psychological insulin resistance has been used as a term to encompass all the barriers to initiating insulin [22-24]. In our study we distinguished this as a single category separate from other categories but recognized that the range of characteristics within this single category is broad, including social stigma, feelings of personal failure and self blame, feelings of inconvenience, and fear of adverse effects from insulin. A cultural barrier to initiating insulin therapy has been reported among Chinese living in Canada [15]. For example, injection treatments are reserved for severe conditions in Chinese culture. Therefore, Chinese would prefer traditional medications before accepting insulin therapy. Social stigma also includes the fear of being recognized as being a drug addict. Many patients view the need for insulin as evidence of personal failure or self blame for requiring insulin [21,22].

Some of our patients mentioned fear of hypoglycemia even if they had not personally experienced insulin therapy. Severe hypoglycemia is a dangerous adverse effect of insulin treatment. Duration of insulin treatment has been confirmed as an independent risk factor for severe hypoglycemia Davis and Renda [23]. Following initiation and during continuation of insulin treatment, avoidance of hypoglycemia should always be the first priority. In addition to hypoglycemia, weight gain and inconvenience will affect adherence to treatment with insulin. New insulin analogues might be used to reduce risk of hypoglycemia and weight gain. Using traditional insulin syringes in public may result in social embarrassment and social rejection. Pen injectors, compared to syringes, are easier to use and might lessen inconvenience and thus improve in adherence [12,16]. Because patients and physicians are hesitant about starting insulin treatment, rates of insulin treatment are low in Taiwan. Although decision-making about treatment is made by considering all alternative therapies, decisions are adversely affected by erroneous information and underlying psychological fear or anxiety. The reluctant attitude of physicians to insulin treatment might also influence patients to overlook their problem and work against patient empowerment.

To overcome barriers, establishment of a therapeutic alliance and shared decision-making model between physicians and patients is recommended. The shared decision-making model, compared with paternalism and informed decision making model, is interactional, fosters better communication, provides more care, and is more personal and more comfortable to patients. The shared decision-making model has been advocated to be an ideal model for treatment decision making in the medical encounter. A shared decision model was also reported to be helpful in adherence to insulin treatment [25-27].

A limitation of this study is the sampling method. Patients might refuse the interview if they had an unshakable mind. For example, we had patients with needle phobia who insisted on no interview because they thought insulin injection was impossible for them anyway. The people with low adaptation capacity might have refused the interview because of their dependent lifestyle. We had more women than men 
Citation: Chen KW, Tseng HM, Huang YY, Chuang YJ (2012) The Barriers to Initiating Insulin Therapy among People with Type 2 Diabetes in Taiwan - A Qualitative Study. J Diabetes Metab 3:194. doi:10.4172/2155-6156.1000194

agreeing to the interview. However, the interviewers were experienced and skilled in establishing a good relationship with patients. We also had patients who were willing to talk about their thoughts and feelings. Therefore we believe that we obtained enough information to develop the themes we have described.

\section{Conclusion}

The barriers to initiating insulin treatment among Taiwanese are highlighted in this qualitative study. The decisions are deviated by these barriers: 1) reluctance of physicians 2) misconceptions about insulin 3) low adaptation capacity 4) needle phobia and 5) psychological insulin resistance. Based upon our observations, we could develop better strategies to break these barriers. We should put more responsibility of insulin treatment on caregivers for people with low adaptation capacity. People with needle phobia should be managed as having a disease by desensitizing the phobia. The benefits of early insulin treatment should be included as early as possible in diabetes education. Physicians should adhere to guidelines to initiate insulin treatment and empower patients. Establishment of a therapeutic alliance and a shared decision-making model are fundamental to breaking barriers to insulin treatment and thus improve initiation of and adherence to insulin treatment.

\section{Acknowledgement}

This research was supported by the grant of St. Paul's Hospital SPMRPU1-5001. The authors would like to thanks to Ms. Yu-Ting Ye and Meng-Han Shih, the diabetes educators of the diabetes center at Linko Chang Gung Memoria Hospital and Ms. Huang-Chun Yeh of St. Paul's Hospital. We are also grateful to Professor Wilfred Y. Fuji motor, University of Washington, for his review of this manuscript.

\section{References}

1. (1993)The effect of intensive treatment of diabetes on the development and progression of long-term complications in insulin-dependent diabetes mellitus. The Diabetes Control and Complications Trial Research Group. N Engl J Med 329: 977-986.

2. (1998)Intensive blood-glucose control with sulphonylureas or insulin compared with conventional treatment and risk of complications in patients with type 2 diabetes (UKPDS 33). UK Prospective Diabetes Study (UKPDS) Group. Lance 352: 837-853.

3. American Diabetes Association (2005) Standards of medical care in diabetes. Diabetes Care 28: S4-S36.

4. Weng J, Li Y, Xu W, Shi L, Zhang Q, et al. (2008) Effect of intensive insulin therapy on beta-cell function and glycaemic control in patients with newly diagnosed type 2 diabetes: a multicentre randomised parallel-group trial. Lancet 371: $1753-1760$

5. Alvarsson M, Sundkvist G, Lager I, Berntorp K, Fernqvist-Forbes E, et al. ( 2008) Effects of insulin vs. glibenclamide in recently diagnosed patients with type 2 diabetes: a 4-year follow-up. Diabetes Obes Metab 10: 421-429.

6. Yoshioka K, Yoshida T, Yoshikawa T (2004) Short-term intensive insulin therapy in newly diagnosed type 2 diabetes: response to Ryan, Imes, and Wallace. Diabetes Care 27: 2281-2282.

7. Ilkova H, Glaser B, Tunckale A, Bagriacik N, Cerasi E (1997) Induction of longterm glycemic control in newly diagnosed type 2 diabetic patients by transient intensive insulin treatment. Diabetes Care 20: 1353-1356.

8. Hoerger TJ, Ahmann AJ (2008) The impact of diabetes and associated cardiometabolic risk factors on members: strategies for optimizing outcomes. J Manag Care Pharm 14: S2-S14.

9. Yu NC, Su HY, Tsai ST, Lin BJ, Shiu RS, et al. (2009) ABC control of diabetes: survey data from National Diabetes Health Promotion Centers in Taiwan. Diabetes Res Clin Pract 84: 194-200.

10. Korytkowski M (2002) When oral agents fail: practical barriers to starting insulin. Int J Obes Relat Metab Disord 3: S18-S24.

11. Larkin ME, Capasso VA, Chen CL, Mahoney EK, Hazard B, et al. (2008) Mea- suring psychological insulin resistance: barriers to insulin use. Diabetes Educ 34: $511-517$

12. Magwire ML (2011) Addressing Barriers to Insulin Therapy: The Role of Insulin Pens. Am J Ther 18: 392-402.

13. Meece J (2006) Dispelling myths and removing barriers about insulin in type 2 diabetes. Diabetes Educ 32: 9S-18S.

14. Peyrot M, Rubin RR, Khunti K (2010) Addressing barriers to initiation of insulin in patients with type 2 diabetes. Prim Care Diabetes 1: S11-S18.

15. Ho EY, James J (2006) Cultural Barriers to Initiating Insulin Therapy in Chinese People With Type 2 Diabetes Living in Canada. Can J Diabetes 30: 390 -396.

16. Brunton S (2008) Initiating insulin therapy in type 2 diabetes: benefits of insulin analogs and insulin pens. Diabetes Technol Ther 10: 247-256.

17. Ritchie J, Spencer E (1994) Qualitative data analysis for applied policy research.

18. Col N, Fanale JE, Kronholm P (1990) The role of medication noncompliance and adverse drug reactions in hospitalizations of the elderly. Arch Intern Med 150: 841-845.

19. Wallace TM, Matthews DR (2000) Poor glycaemic control in type 2 diabetes: a conspiracy of disease, suboptimal therapy and attitude. QJM 93: 369-374.

20. Hamilton JG (1995) Needle phobia: a neglected diagnosis. J Fam Pract 41 169-175.

21. Peyrot M, Rubin RR, Lauritzen T, Snoek FJ, Matthews DR, et al. (2005) Psychosocial problems and barriers to improved diabetes management: results of the Cross-National Diabetes Attitudes, Wishes and Needs (DAWN) Study. Diabet Med 22: 1379-1385

22. Polonsky WH, Fisher L, Guzman S, Villa-Caballero L, Edelman SV (2005) Psychological insulin resistance in patients with type 2 diabetes: the scope of the problem. Diabetes Care 28: 2543-2545.

23. Davis SN, Renda SM (2006) Psychological insulin resistance: overcoming barriers to starting insulin therapy. Diabetes Educ 32: 146S-152S.

24. Nam S, Chesla C, Stotts NA, Kroon L, Janson SL (2010) Factors associated with psychological insulin resistance in individuals with type 2 diabetes. Diabetes Care 33: 1747-1749.

25. Karter AJ, Subramanian U, Saha C, Crosson JC, Parker MM, et al. (2010) Barriers to insulin initiation: the translating research into action for diabetes insulin starts project. Diabetes Care 33: 733-735.

26. Charles C, Whelan T, Gafni A (1999) what do we mean by partnership in mak ing decision about treatment? BMJ 319: 780-782.

27. King JS, Eckman MH, Moulton BW (2011) The potential of shared decision making to reduce health disparities. J Law Med Ethics 39: 30-33. 